\title{
A Modified Capacitor Current Feedback Active Damping Approach for Grid Connected Converters with an $L C L$ Filter
}

\author{
Zhiqiang Wan ${ }^{\dagger}$, Jian Xiong ${ }^{*}$, Ji Lei ${ }^{*}$, Chen Chen ${ }^{*}$, and Kai Zhang ${ }^{*}$ \\ ${ }^{\dagger}{ }^{*}$ State Key Laboratory of Advanced Electromagnetic Engineering and Technology, Huazhong University of \\ Science and Technology, Wuhan, China
}

\begin{abstract}
Capacitor current feedback active damping is extensively used in grid-connected converters with an LCL filter. However, systems tends to become unstable when the digital control delay is taken into account, especially in low switching frequencies. This paper discusses this issue by deriving a discrete model with a digital control delay and by presenting the stable region of an active damping loop from high to low switching frequencies. In order to overcome the disadvantage of capacitor current feedback active damping, this paper proposes a modified approach using grid current and converter current for feedback. This can expand the stable region and provide sufficient active damping whether in high or low switching frequencies. By applying the modified approach, the active damping loop can be simplified from fourth-order into second-order, and the design of the grid current loop can be simplified. The modified approach can work well when the grid impedance varies. Both the active damping performance and the dynamic performance of the current loop are verified by simulations and experimental results.
\end{abstract}

Key words: Active damping, Capacitor current feedback, $L C L$ filter

\section{INTRODUCTION}

Nowadays, the number of distributed power generation systems (DPGS) connected to the grid is increasing rapidly. As a result, stricter standards for power quality have been issued [1]. The grid-connected converter plays a crucial part in injecting high quality power into the grid. Due to the $L C L$ grid filters' good filtering characteristic above resonance frequency, they are increasingly being used to mitigate PWM harmonics, especially in high power DPGSs whose switching frequency is limited by losses [2]. Unfortunately, the inherent resonance of the $L C L$ filter can cause undesired oscillations or even instasbility. A simple method to damp this resonance is to add a passive resistor to the $L C L$ filter [3]. However, this is not appropriate for high power DPGSs due to their power loss [4].

Many active damping approaches have been proposed

Manuscript received Feb. 13, 2015; accepted Apr. 22, 2015

Recommended for publication by Associate Editor Bon-Gwan Gu.

†Corresponding Author: wanzhiqiang1990@126.com

Tel: +86-13260589905

* State Key Laboratory of Advanced Electromagnetic Engineering and Technology, Huazhong University of Science and Technology, China which are more flexible and efficient than the passive damping techniques [5]-[8]. Capacitor current feedback active damping has been extensively used because it is simple to realize among the various active damping approaches [9]-[11]. However, in a digitally controlled system, there is an inevitable digital control delay between the sampling instant and the PWM updating instant which is equivalent to applying a unit time delay $z^{-1}$ in the control loop [12]-[14]. Few references have thoroughly discussed the stability and damping performance of capacitor current feedback active damping when the digital control delay is taken into account, especially at low switching frequencies. Reference [15] totally neglects the influence of digital control delay. As its switching frequency as high as $10 \mathrm{kHz}$, the influence is not obvious. Digital control delay is regarded as a first-order inertia in reference [16] which is suitable for its application with a $5 \mathrm{kHz}$ switching frequency. However, in high power systems, the switching frequency is limited by power losses and cannot be set very high. Furthermore, the above references design the active damping loop in the continuous time domain. However, this will lead to system instability and poor damping performance in low switching frequencies 


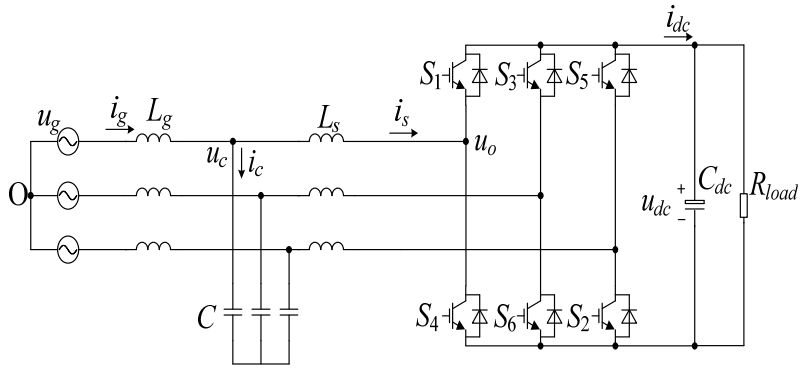

Fig. 1. Grid connected PWM converter with $L C L$ filter.

such as $2 \mathrm{kHz}$. Reference [17] demonstrates the stable region of active damping in the discrete time domain when the digital control delay is taken into account. However, it only presents the stable region in the $5 \mathrm{kHz}$ switching frequency. When the switching frequency decreases, the system becomes inherently unstable no matter how the capacitor current feedback coefficient changes. Therefore, it is unsuitable to neglect the digital control delay at low switching frequencies when designing the active damping loop. This paper addresses this issue by theoretically presenting the stable regions of capacitor current feedback active damping in high, medium and low switching frequencies.

In high power applications, both the grid current and the converter current are sensed in the $L C L$ filter system. The grid current is used in current loop control, and the converter current is used to protect IGBTs from overcurrent. The capacitor current can be calculated by the grid current minus the converter current. This means that the capacitor current feedback is equal to the grid current and converter current feedback with the same feedback coefficient which will lose one degree of control freedom. In order to increase the degree of control freedom and to expand the stable region, especially at low switching frequencies, this paper proposes a modified active damping approach with grid current and converter current feedback which can strengthen system stability and provide sufficient active damping. Moreover, the modified approach does not need any additional sensors. When the grid impedance varies, the modified approach can also work well. Simulation and experimental results based on a $300 \mathrm{kVA}$ three phase prototype verify the theoretical conclusions presented in this paper.

\section{MODELING OF THE LCL FILTER}

A diagram of a grid connected PWM converter with an $L C L$ filter is shown in Fig. 1, where $i_{g}, i_{s}, u_{c}, i_{c}$ and $u_{o}$ represent the grid current, converter current, filter capacitor voltage, filter capacitor current and converter voltage, respectively.

The state space equation of an $L C L$ filter in the continuous time domain yields as Equ. (1) by applying Kirchhoff laws [18], where the state vector is defined as

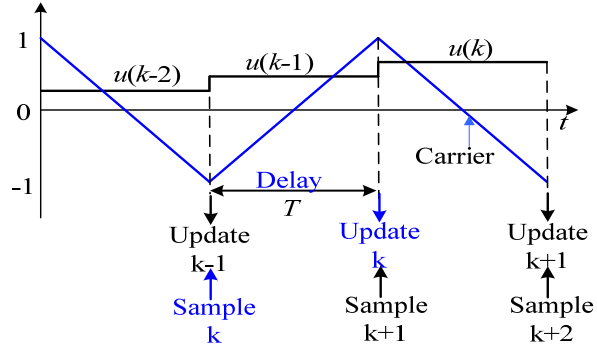

Fig. 2. Digital control delay in digital control system.

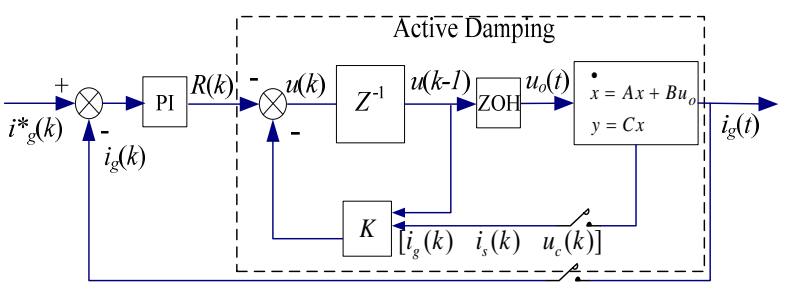

Fig. 3. Control structure with digital control delay.

$$
\begin{aligned}
x(t)=\left[\begin{array}{lll}
i_{g}(t) & i_{S}(t) \quad u_{c}(t)
\end{array}\right]^{\mathrm{T}} & . \\
\dot{x} & =A x+B u_{o} \\
y & =C x
\end{aligned}
$$

The system matrix, input matrix and output matrix are shown as Equ. (2). The parasitic resistors of the inductors are not taken into account in the following derivation.

$$
A=\left[\begin{array}{ccc}
0 & 0 & -\frac{1}{L_{g}} \\
0 & 0 & \frac{1}{L_{S}} \\
\frac{1}{C} & -\frac{1}{C} & 0
\end{array}\right] \quad B=\left[\begin{array}{c}
0 \\
-\frac{1}{L_{S}} \\
0
\end{array}\right] \quad C=\left[\begin{array}{lll}
1 & 0 & 0
\end{array}\right]
$$

In a digital control system, a digital processor needs some time to make calculations [12]. Therefore, the calculated result of the present step is updated to output at the beginning of the next step which is shown in Fig. 2. As a result, there is an inevitable digital control delay between the sampling instant and the PWM updating instant which is equivalent to applying a unit time delay $z^{-1}$ in the control loop. The digital control delay has a crucial influence on system stability and damping performance. It is necessary to take the digital control delay into account when designing an active damping loop, especially at low switching frequencies. In this paper, the sampling instant is located at the bottom and the summit of the carrier. Therefore, the sampling frequency is double the switching frequency.

Fig. 3 shows the grid current control structure with the digital control delay. By active damping, the current loop can be controlled with a PI controller. The acitve damping is achieved by state feedback. Choosing different feedback states corresponds to different active damping approaches. $K$ is the feedback matrix. 


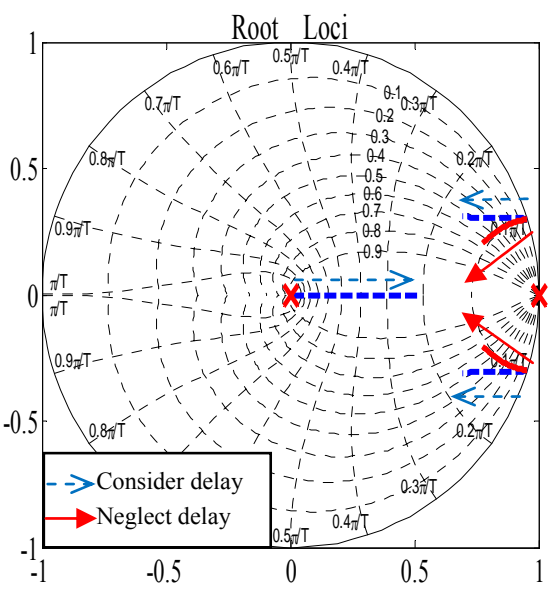

(a) $10 \mathrm{kHz}$ switching frequency.

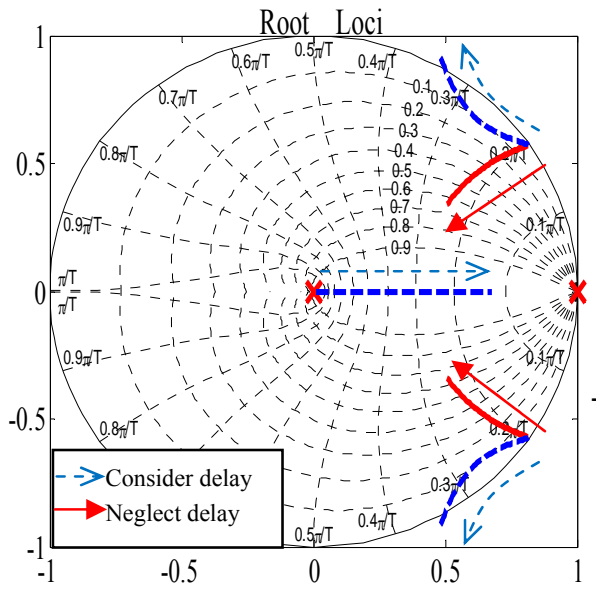

(b) $5 \mathrm{kHz}$ switching frequency.

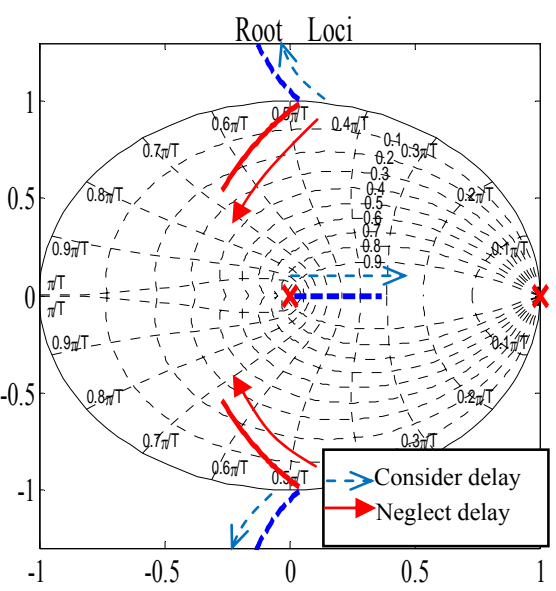

(c) $2 \mathrm{kHz}$ switching frequency.

Fig. 4. Root loci of capacitor current feedback when considering digital control delay and neglecting digital control delay.

The discrete model is derived as Equ. (3), where the state vector is defined as $x(k)=\left[\begin{array}{lll}i_{g}(k) & i_{s}(k) & u_{c}(k)\end{array}\right]^{\mathrm{T}}$.

$$
\begin{aligned}
& x(\mathrm{k}+1)=E x(k)+F u_{o}(k) \\
& y(k)=C x(k)
\end{aligned}
$$

The discrete system matrix and the input matrix can be calculated with:

$$
E=e^{A T} \quad F=\int_{0}^{T} e^{A t} d t B
$$

The systme matrix and the output matrix yield as follows:

$$
E=\left[\begin{array}{ccc}
1-\frac{(1-\cos \omega T) L_{s}}{L_{\mathrm{g}}+L_{s}} & \frac{(1-\cos \omega T) L_{s}}{L_{\mathrm{g}}+L_{s}} & -\frac{\sin \omega T}{\omega L_{g}} \\
\frac{(1-\cos \omega T) L_{g}}{L_{\mathrm{g}}+L_{s}} & 1-\frac{(1-\cos \omega T) L_{g}}{L_{\mathrm{g}}+L_{s}} & \frac{\sin \omega T}{\omega L_{s}} \\
\frac{\sin (\omega T)}{\omega C} & -\frac{\sin (\omega T)}{\omega C} & \cos \omega T
\end{array}\right]
$$

Due to the digital control delay, there is a unit time delay $z^{-1}$ between the reference voltage $u(k)$ and the output voltage $u_{o}(k)[19]$.

$$
u_{o}(k)=u(k-1)
$$

In order to exactly analyze the impact of the digital control delay on system stability and the design of the active damping loop, the state space equation taking the digital control delay into account is derived as Equ. (8), where the state vector is defined as $x(k)=\left[\begin{array}{lllll}i_{g}(k) & i_{s}(k) & u_{c}(k) & u(k-1)\end{array}\right]^{\mathrm{T}} \quad[20]$.

$$
\begin{aligned}
& x(\mathrm{k}+1)=G x(k)+H u(k) \\
& y(k)=C x(k)
\end{aligned}
$$

Whereas:

$$
G=\left[\begin{array}{cccc}
e_{11} & e_{12} & e_{13} & f_{1} \\
e_{21} & e_{22} & e_{23} & f_{2} \\
e_{31} & e_{32} & e_{33} & f_{3} \\
0 & 0 & 0 & 0
\end{array}\right] \quad H=\left[\begin{array}{l}
0 \\
0 \\
0 \\
1
\end{array}\right] \quad C=\left[\begin{array}{l}
1 \\
0 \\
0 \\
0
\end{array}\right]^{\mathrm{T}}
$$

with $e_{i j}$ and $f_{i}(i, j \in\{1,2,3\})$ being entries in matrices $E$ and F.

The transfer function of the active damping loop $I_{g}(\mathrm{z}) / R(\mathrm{z})$ can be calculated by equation Equ. (10), where $K$ is the feedback matrix and $I$ is the unit matrix. The following discussions are based on this transfer function.

$$
\frac{I_{g}(z)}{R(z)}=-C \cdot(z \cdot I-G+H \cdot K)^{-1} \cdot H
$$

\section{STABILITY ANALYSIS OF THE CAPACITOR CURRENT FEEDBACK ACTIVE DAMPING}

Based on the discrete model in section II, the influence of the digital control delay on stability and damping performance is discussed in this section. The grid current and converter current are both sensed. The capacitor current is calculated by the grid current minus the converter current. As a result, the feedback matrix is $K=\left[\begin{array}{llll}K_{i c} & -K_{i c} & 0 & 0\end{array}\right]$, where $K_{i c}$ represents the capacitor current feedback coefficient. The capacitor voltage is not sensed so its feedback coefficient is 0 . The transfer function of the active damping loop yields as Equ. (11) by substituting feedback matrix $K$ into Equ. (10). This shows that an active damping loop with the digital control delay has four poles. Three of them move as the feedback coefficient changes. The other is located at $z=1$. 
TABLE I

PARAMETER OF LCL FILTER

\begin{tabular}{ccc}
\hline Symbol & Quantity & Value \\
\hline$L_{g}$ & Grid inductance & $90 \mu \mathrm{H}(6 \%)$ \\
$L_{s}$ & Converter inductance & $180 \mu \mathrm{H}(12 \%)$ \\
$C$ & Filter capacitor & $450 \mu \mathrm{F}(6.8 \%)$ \\
$\omega$ & Resonance frequency & $969 \mathrm{~Hz}$ \\
\hline
\end{tabular}

$\frac{I_{g}(z)}{R(z)}=\frac{\omega T\left[z^{2}-2 z \cos (\omega T)+1\right]-(z-1)^{2} \sin \omega T}{L_{s} L_{g} C \omega^{3}(z-1)\left[z^{3}+2 \cos (\omega T) z^{2}+\left(\frac{K_{i c}}{\omega L_{s}} \sin \omega T+1\right) z-\frac{K_{i c}}{\omega L_{s}} \sin \omega T\right]}$

Fig. 4 shows the root loci of the capacitor current feedback active damping loop. This demonstrates the stable regions both while considering the digital control delay (dashed line) and while neglecting the digital control delay (solid line) in high $(10 \mathrm{kHz})$, medium $(5 \mathrm{kHz})$ and low $(2 \mathrm{kHz})$ switching frequencies with parameters given in Table $\mathrm{I}$.

The active damping loop without the digital control delay also has four poles. Two of them move as the feedback coefficient changes. The others are located at $z=1$ and $z=0$, respectively. When the digital control delay is not taken into account, the system is always stable. However, a system with the digital control delay tends to become unstable, especially at low switching frequencies.

Fig. 4(a) demonstrates the root loci of a high $(10 \mathrm{kHz})$ switching frequency. The damping performance of the dashed line (considering the delay) is nearly the same as that of the solid line (neglecting the delay). Therefore, the influence of the digital control delay is not obvious in high switching frequencies.

Fig. 4(b) demonstrates the root loci of a medium $(5 \mathrm{kHz})$ switching frequency. The damping ratio of the dashed line (considering the delay) is much smaller than that of the solid line (neglecting the delay). It is easy for the system to become resonant or even unstable at a medium switching frequency when considering the digital control delay.

Fig. 4(c) demonstrates the root loci of a low $(2 \mathrm{kHz})$ switching frequency. The damping performance of the dashed line (considering the delay) is different from that of the solid line (neglecting the delay). A system with the digital control delay is inherently unstable no matter how the feedback coefficient changes at a low switching frequency.

\section{MOdIFIEd ACTIVE DAMPING APPROACH}

As discusses in section III, the capacitor current feedback is equal to the grid current and converter current feedback with the same feedback coefficient which loses one degree of control freedom. This is unsuitable at a low switching frequency. In order to increase the degree of control freedom, this paper sets different feedback coefficients for the grid current and converter current which strengthens the system stability. Moreover, the modified approach does not need additional sensors. Therefure, the feedback matrix is $K=\left[\begin{array}{llll}k_{i g} & k_{i s} & 0 & k_{u}\end{array}\right]$. Where, $k_{i g}$ and $k_{i s}$ represent the feedback coefficients of the grid current and the converter current, respectively. The capacitor voltage is not sensed so its feedback coefficient is $0 . k_{u}$ represents the feedback coefficient of the reference voltage $u(k-1)$.

The transfer function of the active damping loop $I_{g}(\mathrm{z}) / R(\mathrm{z})$ is derived by substituting feedback matrix $K$ and the parameters of TABLE I into Equ. (10). The characteristic equation of the active damping loop is shown as Equ. (12).

$$
\begin{aligned}
z^{4} & +\left(k_{u}-1.1\right) z^{3}+\left(-0.32 k_{i g}-1.23 k_{i s}-1.1 k_{u}+1.1\right) z^{2} \\
& +\left(-1.12 k_{i g}+0.7 k_{i s}+1.1 k_{u}-1\right) z+\left(-0.32 k_{i g}-1.23 k_{i s}-k_{u}\right)=0
\end{aligned}
$$

This shows that the active damping loop has four poles. Assume that the desired poles are $p_{1}, p_{2}, p_{3}, p_{4}$, where $p_{3}, p_{4}$ represent the pair of conjugate complex poles. Therefore, the desired characteristic equation is derived as Equ. (13).

$$
z^{4}+a_{3} z^{3}+a_{2} z^{2}+a_{1} z+a_{0}=0
$$

Where:

$$
\left\{\begin{array}{l}
a_{3}=-p_{1}-p_{2}-p_{3}-p_{4} \\
a_{2}=p_{1} p_{2}+p_{1} p_{3}+p_{1} p_{4}+p_{2} p_{3}+p_{2} p_{4}+p_{3} p_{4} \\
a_{1}=-p_{1} p_{2} p_{3}-p_{1} p_{2} p_{4}-p_{1} p_{3} p_{4}-p_{2} p_{3} p_{4} \\
a_{0}=p_{1} p_{2} p_{3} p_{4}
\end{array}\right.
$$

Expressions for the feedback coefficients in terms of the desired pole locations are obtained by comparing Equ. (12) with Equ. (13). The equations are derived as Equ. (15).

$$
\left\{\begin{aligned}
k_{u}-1.1 & =a_{3} \\
-0.32 k_{i g}-1.23 k_{i s}-1.1 k_{u}+1.1 & =a_{2} \\
-1.12 k_{i g}+0.70 k_{i s}+1.1 k_{u}-1 & =a_{1} \\
-0.32 k_{i g}-1.23 k_{i s}-k_{u} & =a_{0}
\end{aligned}\right.
$$

In linear algebra, the solvable condition of the equation $A \cdot X=b$ is that $\operatorname{Rank}(A \mid b)=\operatorname{Rank}(A)=$ number of variable [21]. In Equ. (15), $\operatorname{Rank}(A)=$ number of variable=3 . Therefore, the solvable condition of Equ. (15) is $\operatorname{Rank}(A \mid b)=3$. Use Gaussian Elimination to transform the augmented matrix $A \mid b$ to an upper triangular matrix as Equ. (16).

$$
A \mid b=\left[\begin{array}{cccc}
-0.32 & -1.23 & -1.1 & -1.1+a_{2} \\
0 & 5.05 & 4.98 & 4.88+a_{1}-3.54 \cdot a_{2} \\
0 & 0 & 1 & 1.1+a_{3} \\
0 & 0 & 0 & 0.99+a_{0}-a_{2}-0.1 \cdot a_{3}
\end{array}\right]
$$

When the fourth row of the augmented matrix $A \mid b$ is equal to $0, \operatorname{Rank}(A \mid b)=3$. Therefore, the solvable condition of Equ. (15) is derived as Equ. (17).

$$
0.99+a_{0}-a_{2}-0.1 \cdot a_{3}=0
$$

The desired poles must satisfy Equ. (17). In order to derive the root loci of the active damping loop in the discrete domain, set the dominant pole $p_{1}=0.9$, and the non-dominant pole $p_{2}=0.1$, and assume the pair of conjugate complex poles 


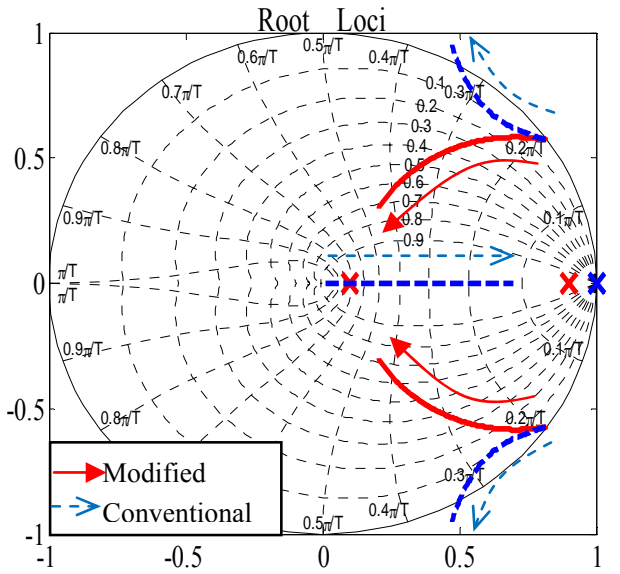

(a) $5 \mathrm{kHz}$ switching frequency.

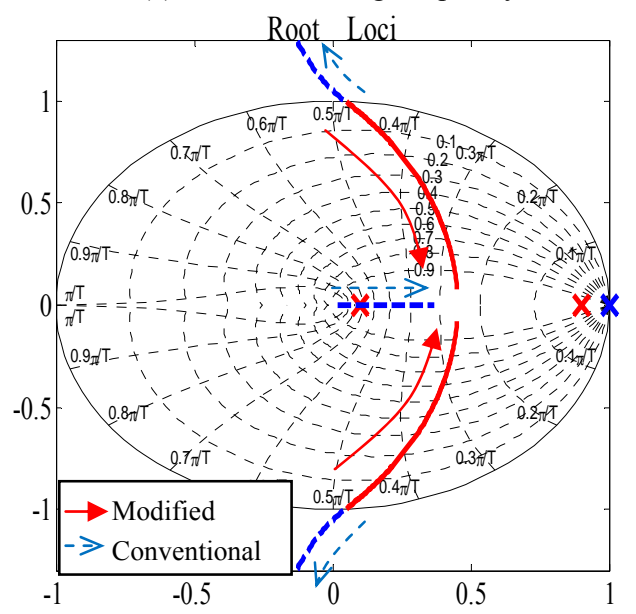

(b) $2 \mathrm{kHz}$ switching frequency.

Fig. 5. Root loci of active damping loop comparing modified approach with conventional capacitor current feedback.

to be $p_{3}=\alpha+j \cdot \beta$, and $p_{4}=\alpha-j \cdot \beta$. Substituting $p_{1}, p_{2}, p_{3}, p_{4}$ into Equ. (17) yields the equation of the conjugate complex poles as Equ. (18).

$$
-0.91 \cdot \alpha^{2}-0.91 \cdot \beta^{2}-1.80 \cdot \alpha+1=0
$$

The root loci of the active damping loop, comparing modified approach with the conventional capacitor current feedback active damping, is shown in Fig. 5.

Fig. 5(a) shows the root loci of a medium $(5 \mathrm{kHz})$ switching frequency. The modified approach (solid line) can provide a larger damping ratio than the conventional capacitor current feedback active damping (dashed line) in a medium switching frequency.

Fig. 5(b) shows the root loci of a low $(2 \mathrm{kHz})$ switching frequency. The conventional capacitor current feedback active damping (dashed line) is unstable. However, the modified approach (solid line) can overcome this issue at a low switching frequency.

When compared with the conventional capacitor current feedback, the modified approach can strengthen stability and provide sufficient damping performance, especially in low

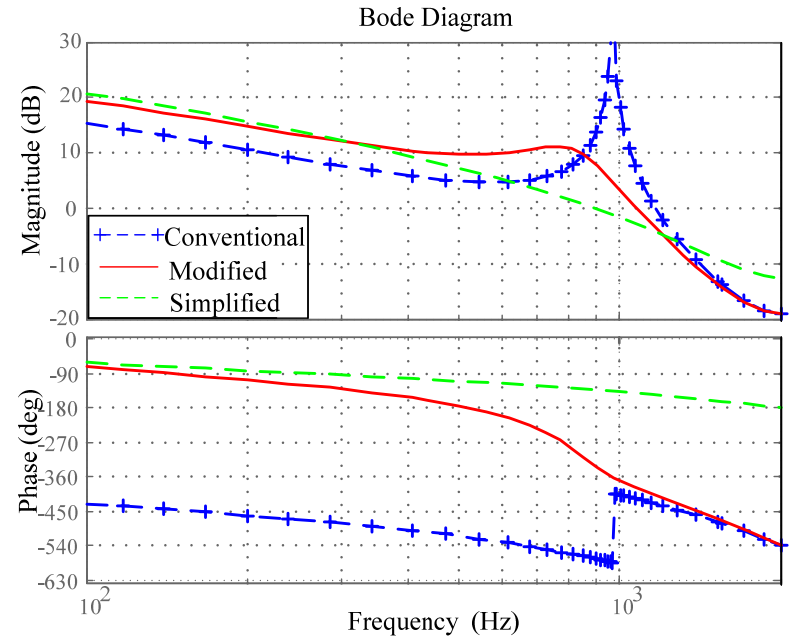

Fig. 6. Bode diagram of active damping loop comparing modified approach with conventional capacitor current feedback in $2 \mathrm{kHz}$ switching frequency.

switching frequencies. Choosing proper locations of the desired poles $p_{1}, p_{2}, p_{3}, p_{4}$ and substituting them into Equ. (15), the feedback matrix $K$ can be solved out.

Fig. 6 shows a bode diagram of the active damping loop, comparing the modified approach with the conventional capacitor current feedback approach, at a $2 \mathrm{kHz}$ switching frequency. It demonstrates that the conventional capacitor current feedback (marked line) causes resonance. By applying the modified approach (solid line), the resonance can be damped.

By applying the modified approach, the pair of conjugate complex poles $p_{3}, p_{4}$ can be placed away from the dominant pole $p_{1}$. Therefore, the conjugate complex poles have little influence on the current loop in the low frequency band. The cut off frequency of the current loop is always set to the low frequency band so that it is suitable to neglect the conjugate complex poles when designing the current loop [22].

By neglecting the pair of conjugate complex poles, the active damping loop is simplified from fourth-order into second-order. This will simplify the design of the current loop. Fig. 6 demonstrates that dashed line (neglecting the conjugate complex poles) is nearly the same as the solid line (considering the conjugate complex poles) in the low frequency band.

Variations of the grid impedance in weak grids has an impact on active damping performance [23]. Therefore, it is necessary to do a sensitivity analysis when the grid impedance is taken into account. Fig. 7 shows the root loci of the active damping loop when the grid impedance varies from $0 \mu \mathrm{H}$ to $225 \mu \mathrm{H}$ which is equal to $15 \% \mathrm{pu}$. The conjugate complex poles move towards the circle center, and the other two poles just move a little on the real axis.

Fig. 8 shows a bode diagram of the active damping loop comparing no grid impedance with a $15 \%$ pu grid impedance. It demonstrates that the bode diagram with no grid impedance 


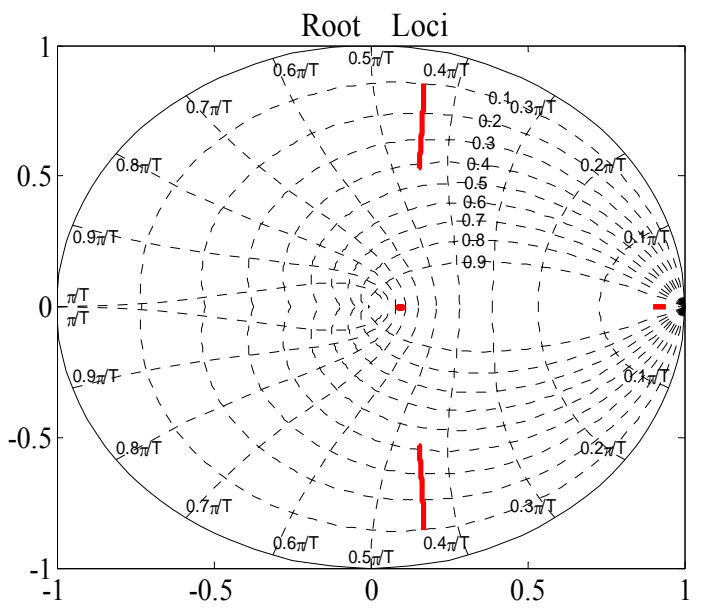

Fig. 7. Root loci of active damping loop when grid impedance varies from $0 \mu \mathrm{H}$ to $225 \mu \mathrm{H}(15 \% \mathrm{pu})$.

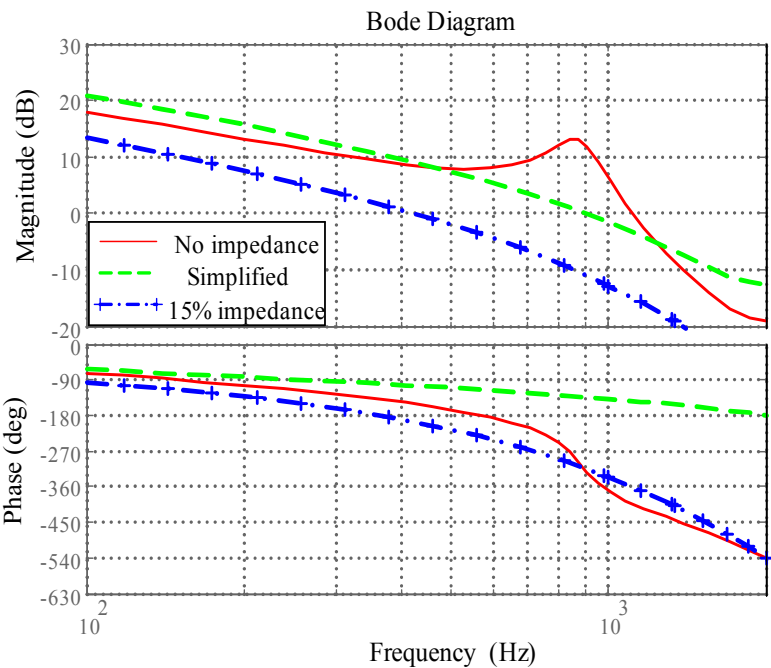

Fig. 8. Bode diagram of active damping loop comparing no grid impedance with $15 \%$ pu grid impedance.

(solid line) is nearly the same the one with a $15 \%$ pu grid impedance (marked line) in the low frequency band. Moreover, the damping performance of the $15 \%$ pu grid impedance is better than that of the no grid impedance. This also demonstrates that the dashed line (neglecting the conjugate complex poles) is nearly the same as with the marked line (15\% pu grid impedance). Therefore, it can be seen that the modified active damping method works well when the grid impedance varies.

\section{SIMULATION AND EXPERIMENT RESULTS}

The analysis is verified with MATLAB and a $300 \mathrm{kVA}$ three phase prototype. The prototype is shown in Fig. 9. The system parameters are given in Table II.

The active damping performance is verified by reactive current reference steps from 0 to $-500 \mathrm{~A}$. The conventional capacitor current feedback simulation results are unstable at a
TABLE II

SYSTEM PARAMETER

\begin{tabular}{ccc}
\hline Symbol & Quantity & Value \\
\hline$U_{g}$ & Grid voltage(line, rms) & $380 \mathrm{~V}$ \\
$f_{g}$ & Grid frequency & $50 \mathrm{~Hz}$ \\
$U_{D C}$ & DC link voltage & $750 \mathrm{~V}$ \\
$P_{n}$ & Nominal power & $300 \mathrm{kVA}$ \\
$C_{D C}$ & DC link capacitance & $13.8 \mathrm{mF}$ \\
\hline
\end{tabular}

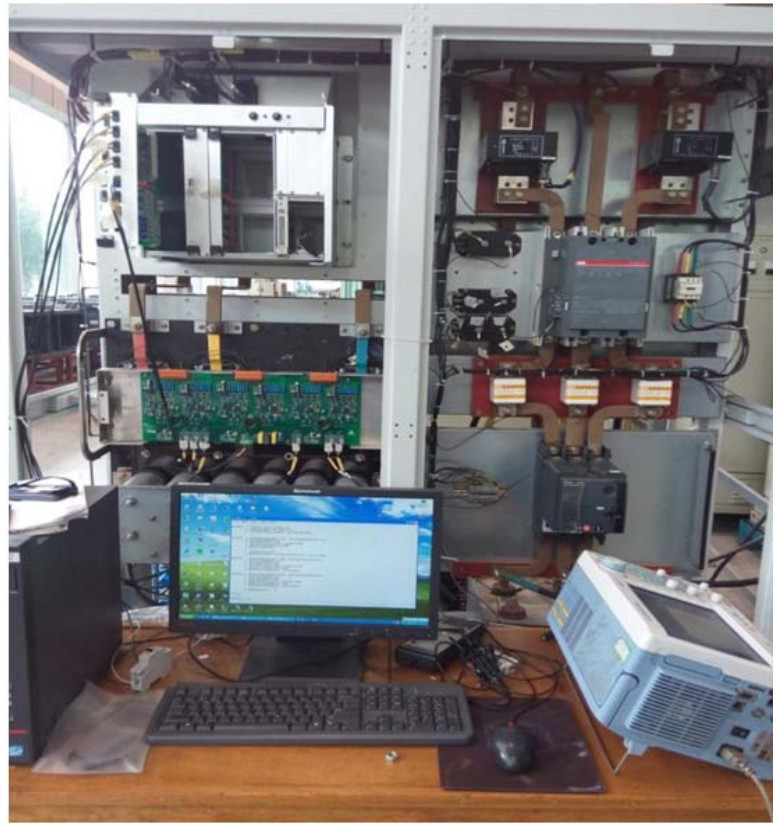

Fig. 9. Prototype of $300 \mathrm{kVA}$ PWM converter.

$2 \mathrm{kHz}$ switching frequency. Fig. 10 shows the results of the modified approach at a $2 \mathrm{kHz}$ switching frequency. Fig. 10(a) shows the simulation results, and Fig. 10(b) shows the experiment results. It can be seen that the system is stable and that the resonance is well damped. The total harmonic distortion (THD) of the grid current is $2.27 \%$ for the simulation and $2.52 \%$ for the experiment. The experimental results match the simulation results well. This demonstrates that the modified approach can strengthen the system stability and provide sufficient damping performance.

Grid current waveforms are obtained by an oscilloscope whose sampling frequency is $500 \mathrm{kHz}$. In order to see the dynamic performance of Fig. 10, the grid current is transformed from the three phase stationary frame into the two phase rotary frame by MATLAB. Fig. 11 shows the step response of the reactive current component. Fig. 11(a) shows the simulation results, and Fig. 11(b) shows the experiment results. There is a one-step delay between the references and the results because of the digital control delay. The experimental results match the simulation results well. The rising time and overshoot are acceptable. This demonstrates that modified approach has good dynamic performance. 

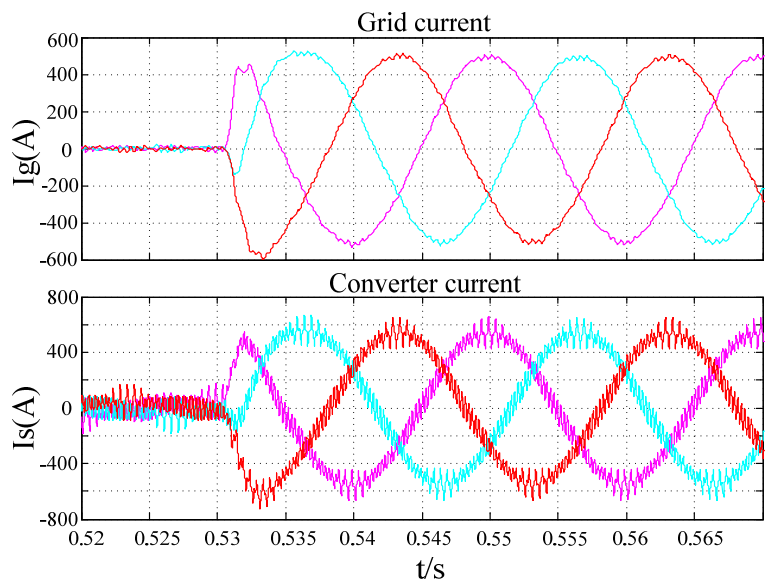

(a) Simulation result of modified approach.

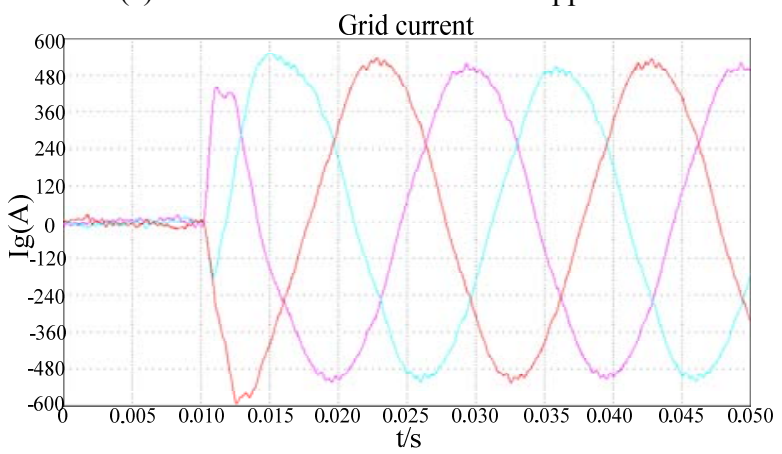

(b) Experiment result of modified approach.

Fig. 10. Reactive current step from 0 to $-500 \mathrm{~A}$ in $2 \mathrm{kHz}$ switching frequency.

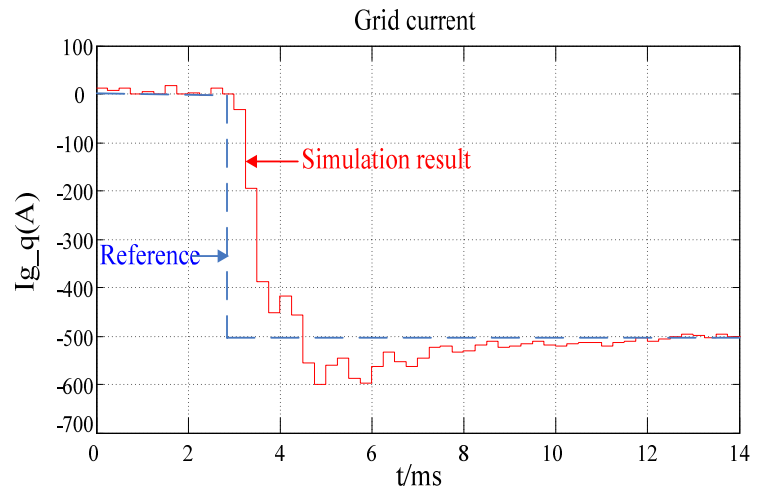

(a) Simulation result of modified approach.

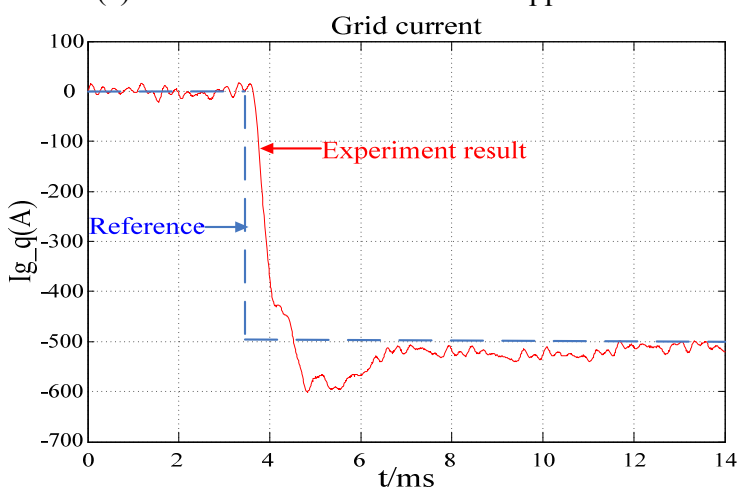

(b) Experiment result of modified approach.

Fig. 11. Step respond of reactive current component from 0 to $-500 \mathrm{~A}$.
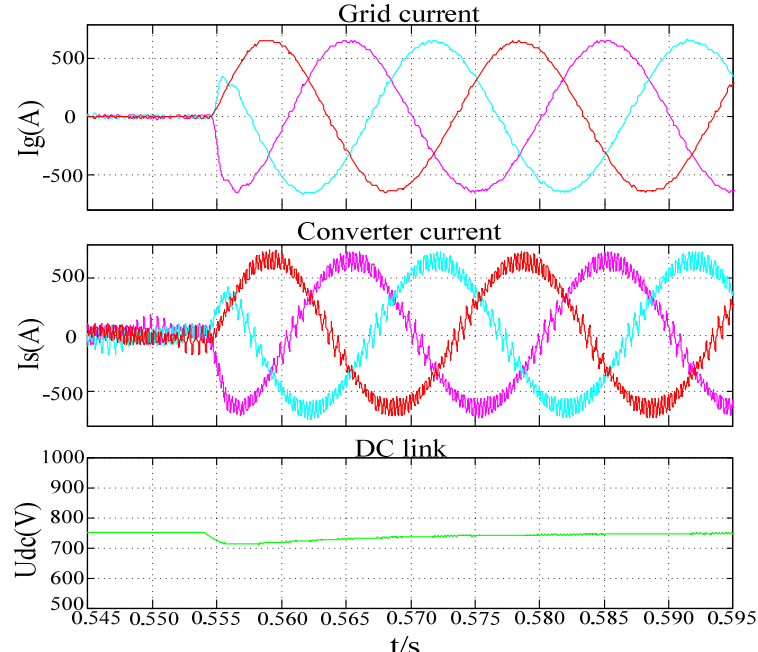

(a) Simulation result of modified approach. Grid current
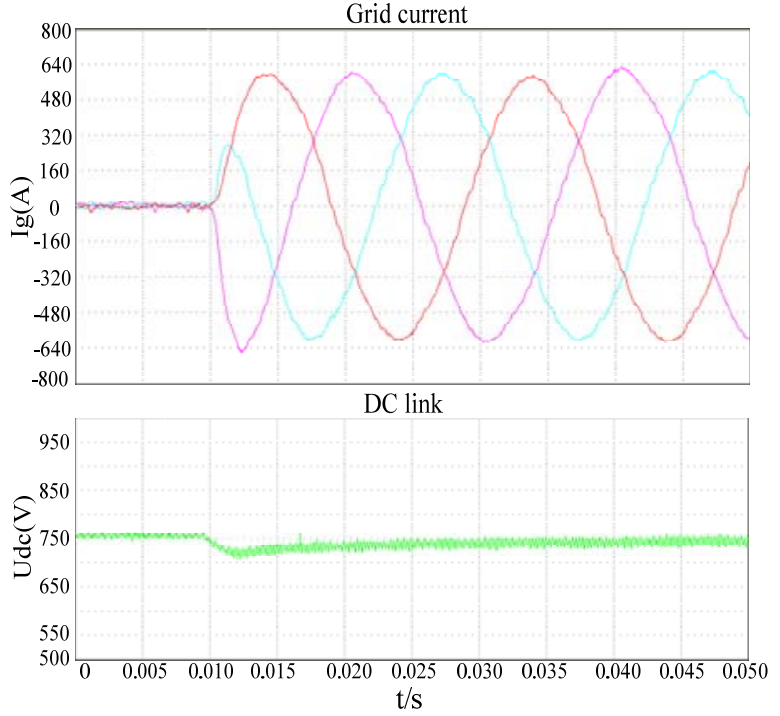

(b) Experiment result of modified approach.

Fig. 12. DC-load step from no-load to full-load in $2 \mathrm{kHz}$ switching frequency.

The test of a DC-load step from no-load to full-load is performed with a $2 \Omega$ resistor which is parallel with a DC link capacitor. Fig. 12 shows the results of the modified approach at a $2 \mathrm{kHz}$ switching frequency. Fig. 12(a) shows the simulation results, and Fig. 12(b) shows the experimental results. The DC bus is $750 \mathrm{~V}$ and the grid current is $600 \mathrm{~A}$ in the steady state.

This demonstrates that the system is stable and that the resonance is well damped. The dynamic respond is fast. The experimental results match the simulation results well.

In order to verify the modified approach when grid impedance is taken into account, a simulation of reactive current stepping from 0 to $-500 \mathrm{~A}$ is done, with the grid impedance set as $225 \mu \mathrm{H}$. Fig. 13 shows the simulation results. The resonance is well damped and the dynamic performance is acceptable. This shows that the modified approach still works well when grid impedance is taken into 


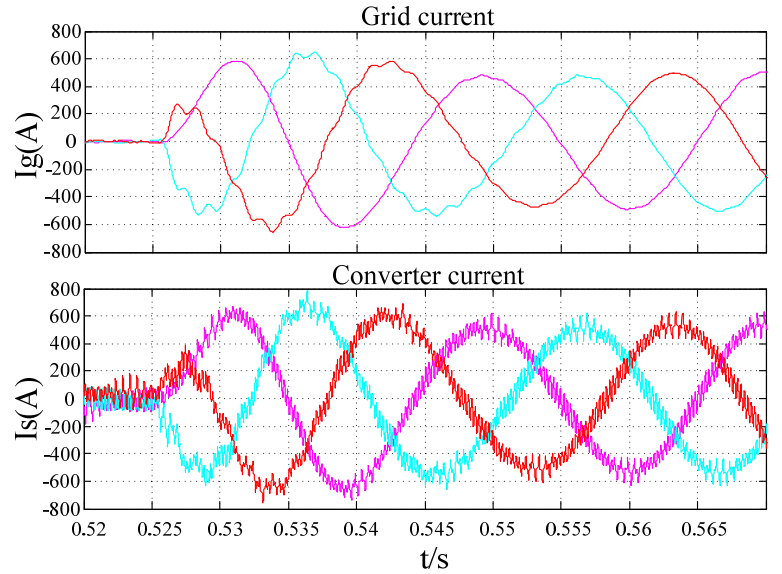

Fig. 13 Reactive current step from 0 to $-500 \mathrm{~A}$ when grid impedance equals $225 \mu \mathrm{H}$.

account.

\section{CONCLUSION}

A modified active damping approach for grid connected PWM converters with a $L C L$ filter has been proposed and analyzed. A discrete model considering the digital control delay has been derived. The feedback coefficients of the modified approach have been yielded in terms of the desired pole locations. The stable regions of the conventional capacitor current feedback active damping and the modified active damping approach have been discussed. The root loci and bode diagram of the active damping loop have been plotted when the grid impedance varies.

This paper shows that it is acceptable for the capacitor current feedback active damping to neglect the digital control delay at a high switching frequency but that systems will become inherent unstable at a low switching frequency. Therefore, it is unsuitable to neglect the digital control delay at a low switching frequency when the designing active damping loop. The modified approach can strengthen system stability and provide sufficient damping performance. By applying the modified approach, the active damping loop can be simplified from fourth-order into second-order and the design of the current loop can be simplified. The modified approach can work well when the grid impedance varies. The theoretical results are verified by simulation and experiment results.

\section{REFERENCES}

[1] F. Blaabjerg, R. Teodorescu, M. Liserre, and A. V. Timbus, "Overview of control and grid synchronization for distributed power generation systems," IEEE Trans. Ind. Electron., Vol. 53, No.5, pp. 1398-1409, Oct. 2006.

[2] K. Jalili and S. Bernet, "Design of LCL filters of active-front-end two-level voltage-source converters," IEEE Trans. Ind. Electron., Vol. 56, No. 5, pp. 1674-1689,
May 2009.

[3] T. C. Y. Wang, Y. Zhihong, S. Gautam, and Y. Xiaoming, "Output filter design for a grid-interconnected three-phase inverter," in Power Electronics Specialist Conference, 2003. PESC '03. 2003 IEEE 34th Annual, Vol. 2, pp. 779-784, 2003.

[4] I. J. Gabe, V. F. Montagner, and H. Pinheiro, "Design and implementation of a robust current controller for VSI connected to the grid through an $L C L$ filter," IEEE Trans. Power Electron., Vol. 24, No. 6, pp. 1444-1452, Jun. 2009.

[5] C. Wessels, J. Dannehl, and F. W. Fuchs, "Active damping of LCL-filter resonance based on virtual resistor for PWM rectifiers ; stability analysis with different filter parameters," in Power Electronics Specialists Conference, 2008. PESC 2008. IEEE, pp. 3532-3538, 2008.

[6] J. Kukkola and M. Hinkkanen, "Observer-based state-space current control for a three-phase grid-connected converter equipped with an LCL filter," IEEE Trans. Ind. Appl., Vol. 50, No.4, pp. 2700-2709, Jul./Aug. 2014.

[7] M. Liserre, A. D. Aquila, and F. Blaabjerg, "Genetic algorithm-based design of the active damping for an LCL-filter three-phase active rectifier," IEEE Trans. Power Electron., Vol. 19, No. 1, pp. 76-86, Jan. 2004.

[8] X. Wang, F. Blaabjerg and P. Loh, "Grid-current-feedback active damping for LCL resonance in grid-connected voltage source converters," IEEE Trans. Power Electron.. to be published.

[9] B. Chenlei, R. Xinbo, W. Xuehua, L. Weiwei, P. Donghua, and W. Kailei, "Design of injected grid current regulator and capacitor-current-feedback active-damping for LCL-type grid-connected inverter," in Energy Conversion Congress and Exposition (ECCE), 2012 IEEE, pp. 579-586, 2012.

[10] M. Wagner, T. Barth, C. Ditmanson, R. Alvarez, and S. Bernet, "Discrete-time optimal active damping of LCL resonance in grid connected converters by proportional capacitor current feedback," in Energy Conversion Congress and Exposition (ECCE), 2013 IEEE, pp. 721-727, 2013.

[11] W. Sun, X. Wu, P. Dai, and J. Zhou, "An over view of damping methods for three-phase PWM rectifier," in Industrial Technology, 2008. ICIT 2008. IEEE International Conference on, pp. 1-5, 2008.

[12] Z. Sanbao and D. Czarkowski, "Modeling and digital control of a phase-controlled series-parallel resonant converter," IEEE Trans. Ind. Electron., Vol. 54, No. 2, pp. 707-715, Apr. 2007.

[13] T. Huu-Phuc, M. F. Rahman, and C. Grantham, "Time delay compensation for a DSP-based current-source converter using observer-predictor controller," in Power Electronics and Drive Systems, 2007. PEDS '07. 7th International Conference on, Bangkok, pp. 1091-1096, 2007.

[14] T. Nussbaumer, M. L. Heldwein, G. Guanghai, S. D. Round, and J. W. Kolar, "Comparison of prediction techniques to compensate time delays caused by digital control of a three-phase buck-type PWM rectifier system," IEEE Trans. Ind. Electron., Vol. 55, No. 2, pp. 791-799, Feb. 2008.

[15] T. Yi, C. L. Poh, W. Peng, H. C. Fook, G. Feng, and F. Blaabjerg, "Generalized design of high performance shunt active power filter with output LCL filter," IEEE Trans. Ind. Electron., Vol. 59, No.3, pp. 1443-1452, Mar. 2012. 
[16] Z. Guohong, T. W. Rasmussen, M. Lin, and R. Teodorescu, "Design and control of LCL-filter with active damping for active power filter," in Industrial Electronics (ISIE), 2010 IEEE International Symposium on, pp. 2557-2562, 2010.

[17] S. Parker, B. McGrath, and G. Holmes, "Regions of active damping control for LCL filters," IEEE Trans. Ind. Appl., Vol. 50, No.1, pp. 424-432, Jan.-Feb. 2014.

[18] J. Dannehl, C. Wessels, and F. W. Fuchs, "Limitations of voltage-oriented PI current control of grid-connected PWM rectifiers with $L C L$ filters," IEEE Trans. Ind. Electron., Vol. 56, No.2, pp. 380-388, Feb. 2009.

[19] P. W. Lehn and M. R. Irvani, "Discrete time modeling and control of the voltage source converter for improved disturbance rejection," IEEE Trans. Power Electron., Vol. 14, No.6, pp. 1028-1036, Nov. 1999.

[20] J. Dannehl, F. W. Fuchs, X. F. Th, and P. B. Gersen, "PI state space current control of grid-connected PWM converters with LCL filters," IEEE Trans. Power Electron., Vol. 25, No.9, pp. 2320-2330, Sep. 2010.

[21] B. Otto, Linear Algebra with Applications, 4th ed. USA: Prentice Hall Publishers, Chap. 1, 2008.

[22] S. Guoqiao, X. Dehong, C. Luping, and Z. Xuancai, “An improved control strategy for grid-connected voltage source inverters with an LCL filter," IEEE Trans. Power Electron., Vol. 23, No. 4, pp. 1899-1906, Jul. 2008.

[23] X. Wang, F. Blaabjerg, and P. Loh, "Virtual RC damping of LCL-filtered voltage source converters with extended selective harmonic compensation," IEEE Trans. Power Electron.. Vol. 30, No. 9, pp. 4726-4737, Sep. 2015.

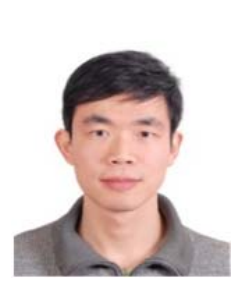

Zhiqiang Wan was born in Jiangxi, China, in 1990. He received his B.S. degree from the Harbin Institute of Technology, Harbin, China, in 2012. He is presently working towards his M.S. degree in the School of Electrical and Electronics Engineering, Huazhong University of Science and Technology (HUST), Wuhan, China. His current research interests include PWM rectifiers, LCL filters and digital control techniques.

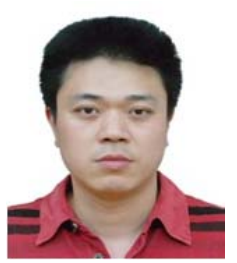

Jian Xiong was born in Hubei Province, China. He received his B.S. degree from the East China Shipbuilding Institute, Zhenjiang, China, in 1993, and his M.S. and Ph.D. degrees from the Huazhong University of Science and Technology (HUST), Wuhan, China, in 1996 and 1999, respectively. He joined HUST as a Lecturer in 1999, and was promoted to an Associate Professor in 2003. His current research interests include uninterruptible power systems, ac drives, switch-mode rectifiers, STATCOMs, and their related control techniques.

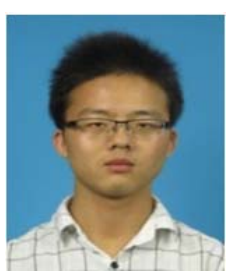

Ji Lei was born in Jiangxi Province, China, in 1991. He received his B.S. degree in Electrical Engineering and Automation from the Hefei University of Technology, Hefei, China, in 2013. He is presently working towards his M.S. degree in Electrical and Electronics Engineering at the Huazhong University of Science and Technology (HUST), Wuhan, China. His current researches include digital control technologies and three-phase unbalance compensation.

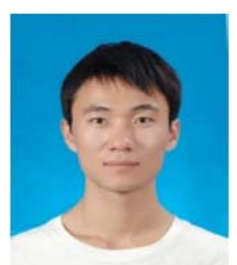

Chen Chen was born in Jiangsu Province, China, in 1991. He received his B.S. degree from Jiangsu University, Zhenjiang, China, in 2012. He is presently working towards his $\mathrm{Ph} . \mathrm{D}$. degree in the School of Electrical and Electronics Engineering, Huazhong University of Science and Technology (HUST), Wuhan, China. His current research interests include digital control techniques, the design and control of power electronics systems, high power factor rectifiers and modular multilevel converters.

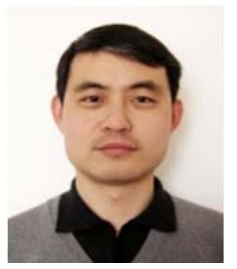

Kai Zhang was born in Henan Province, China. He received his B.S., M.S., and Ph.D. degrees from the Huazhong University of Science and Technology (HUST), Wuhan, China, in 1993, 1996, and 2001, respectively. He joined HUST as an Assistant Lecturer, in 1996. He was a Visiting Scholar at the University of New Brunswick, Saint John, NB, Canada, from 2004 to 2005. He was promoted to a Full Professor in 2006 . He is the author of more than 40 technical papers. His current research interests include uninterruptible power systems, railway traction drives, modular multilevel converters, and electromagnetic compatibility techniques for power electronic systems. 